\title{
The chicken and egg question in excitonic insulators
}

1. Nature of symmetry breaking at the excitonic insulator transition: $\mathrm{Ta}_{2} \mathrm{NiSe}_{5}$ Authors: G. Mazza, M. Rösner, L. Windgätter, S. Latini, H. Hübener, A. J. Millis, A. Rubio, and A. Georges

Phys. Rev. Lett. 124, 197601 (2020).

\section{Nonlinear spectroscopy of collective modes in excitonic insulator} Authors: D. Golež, Z. Sun, Y. Murakami, A. Georges, and A. J. Millis arXiv:2007.09749

\section{Recommended with a Commentary by Atsushi Fujimori, Waseda University}

As the band gap $E_{\mathrm{g}}$ of a semiconductor is reduced to below the exciton binding energy $E_{\mathrm{exc}}$, a macroscopic number of excitons are spontaneously created until the densities of electrons and holes become large enough to screen out the Coulomb attraction between an electron and a hole and the system goes back to a semimetal [1]. Such a state with the collectively formed excitons is called excitonic insulator, in which the excitons condense into a coherent quantum state at low temperature $[2,3]$. While exciton condensation was first observed in bilayer quantum Hall systems [4], there has been continued effort to look for an excitonic insulator in real materials. In particular, the layered transition-metal chalcogenide $1 T$-TiSe2 has been extensively studied, but because the electron pocket and the hole pocket appear at different $\boldsymbol{k}$ points in the Brillouin zone, the resulting possible excitonic state has a finite momentum $\boldsymbol{q}$, i.e., has a spatial modulation. Therefore, the band gap opening in the low-temperature phase of $1 T-\mathrm{TiSe}_{2}$ may be that of an excitonic insulator but also could be due to band folding resulting from the formation of a charge density wave (CDW) or of a phonon softening having the same $\boldsymbol{q}[5]$.

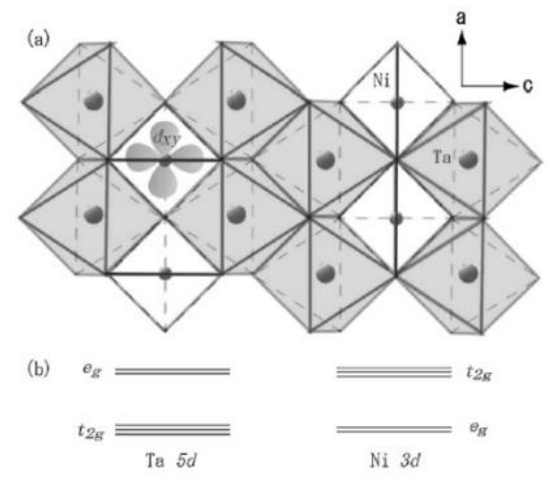

Fig. 1: (a) Structure of $\mathrm{Ta}_{2} \mathrm{NiSe}_{5}$ in the $a b$ plane consisting of two $\mathrm{Ta}$ and one $\mathrm{Ni}$ chains per unit cell running along the $a$ direction. (b) Crystal-field energy levels of the Ta $5 d$ and Ni $3 d$ states (adopted from Ref. 6).

More recently, another layered chalcogenide $\mathrm{Ta}_{2} \mathrm{NiSe}_{5}$ (consisting of $\mathrm{Ta}$ and $\mathrm{Ni}$ chains as shown in Fig. 1) was proposed to be an excitonic insulator [6]. In this compound, both the electron pocket (of the Ta $5 d$ origin) and the hole pocket (of the Ni $3 d$ origin) are located at the 

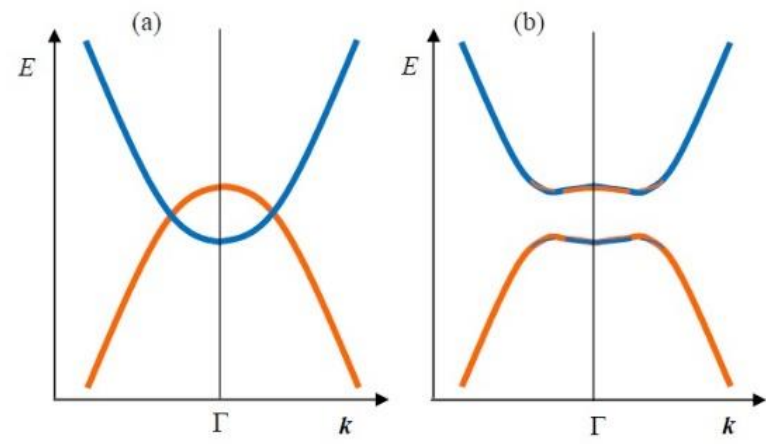

Fig. 2: Schematic band structure of $\mathrm{Ta}_{2} \mathrm{NiSe}_{5}$ for momentum $\boldsymbol{k}$ along the chain direction: (a) Semimetallic state with the unhybridized electron pocket (of Ta $5 d$ origin) and hole pocket (of $\mathrm{Ni} 3 d$ origin) pocket around $\boldsymbol{k}=0$; (b) Gap opening due to hybridization between the electron and hole pockets caused by either excitonic effect or the $\boldsymbol{q}=0$ orthorhombic-tomonoclinic lattice distortion.
Brillouin-zone center and hence $\boldsymbol{q}=0$, as shown in Fig. 2a. ARPES data by Wakisaka et $a l$. [6] exhibited an unusual flattening of the valence-band top (Fig. 2b) below the transition temperature $\left(T_{c} \approx 328 \mathrm{~K}\right)$, as expected for an excitonic insulator. Here, the possibility of CDW is excluded since $\boldsymbol{q}=0$, but the $\boldsymbol{q}=0$ (orthorhombic-to-monoclinic) lattice distortion which occurs below $T_{c}$ [7] may couple to electrons around the Fermi level $\left(E_{\mathrm{F}}\right)$ and may open a gap in a similar way to the excitonic gap. Therefore, whether the band gap of $\mathrm{Ta}_{2} \mathrm{NiSe}_{5}$ primarily originates from excitonic instability $[6,8,9]$ or electron-lattice coupling [10-13] has been the subject of hot debate in recent years. Note that the Ta $5 d$-Ni $3 d$ hybridization at the Brillouin zone center is symmetry-forbidden in the orthorhombic phase, and that either the excitonic instability or the monoclinic distortion

is necessary to induce the hybridization and to open a gap. Note also that, since excitonic effect is a many-body phenomenon, the single-particle picture of Ta $5 d$-Ni $3 d$ hybridization as depicted in Fig. 2b can be drawn after a mean-field treatment of the Ta-Ni inter-site Coulomb interaction.

In order to answer the question of whether the gap opens due to excitonic instability or electron-lattice coupling, a detailed Hartee-Fock study of the realistic multi-band Hubbard model based on first-principles calculation is reported in the first recommended paper by Mazza et al. The main result of the Hartree-Fock calculation for the high-temperature orthorhombic phase is that a spontaneous symmetry breaking of the electronic states occurs into a low-symmetry one which has the same symmetry as that of the low-temperature monoclinic structure. Thus the Ta $5 d$-Ni $3 d$ hybridization becomes allowed in the zone-center region as shown in Fig. 2b. A band gap of $\sim 0.1 \mathrm{eV}$ is opened for a realistic value of inter-site Coulomb interaction. This suggests that the major driving force of the gap opening is of the electronic origin rather than the structural origin. However, it should be noted that, because the electronic and lattice instabilities have the same spatial symmetry, they should appear simultaneously. Therefore, the qualitative question about the origin of the gap above should be replaced by the quantitative question of which of the electronic and structural instabilities is more important to open the gap and to what extent.

Answers to the last question have been given recently by many researchers as follows: Subedi [11] has demonstrated using first-principles theory that the orthorhombic phase has an instability towards the monoclinic structure (through the softening of the " $B_{2 g}$ phonon" at $\boldsymbol{q}=0$ ) and that the band gap opens concomitantly, suggesting the structural origin of the band gap. The strong electron-lattice coupling for the $B_{2} g$ phonon has been confirmed by phonon anomalies observed by Raman scattering [12]. In recent ARPES studies, too, the gap opening has been attributed to Ta $5 d$-Ni $3 d$ hybridization caused by the monoclinic lattice distortion $[10,13]$. 
However, since the electronic and lattice instabilities occur simultaneously and cannot be disentangled by traditional experimental approaches or first-principles calculations, one needs to control the electronic and lattice degrees of freedom separately to answer the quantitative question.

Under such circumstances, a new experimental approach is proposed theoretically by Golež et al. in the second recommended paper: They consider non-linear optical spectroscopy experiment to estimate the relative contributions of the excitonic versus electron-lattice instabilities by probing in-gap optical absorption induced by a pump laser. The in-gap absorption energy is found to sensitively depend on the relative contributions of the collective electronic states and the lattice distortion to the gap opening. We have to wait for such experiment to reach a quantitative and decisive answer. But before that, recent pump-probe ARPES studies on Ta2NiSes by Okazaki et al. [14] and by Tang et al. [15] may give a valuable hint. Immediately (150-300 fs) after an intense infrared-laser pump pulse, the electronic structure was found to transform from the gapped (Fig. 2b) to ungapped semimetallic states (Fig. 2a). On that time scale, the excitonic state was supposed to disintegrate into hot electrons and holes while the lattice would remain monoclinic. If this were the case, the low-temperature monoclinic phase would be semimetallic if the excitonic effect was absent. That is, the gap opening in the low-temperature monoclinic phase would primarily be caused by the excitonic effect and not by the electronlattice coupling.

Although the gap may be formed largely due to the excitonic effect, a coherent excitonic condensate would not be realized, unfortunately, because the minor broken spatial symmetry component would prohibit macroscopic quantum phenomena, as stated by Mazza et al. Since the spatial symmetry breaking in the electronic state is strongly coupled to and even enhanced by the lattice distortion, if one can reduce the lattice distortion, e.g., by applying pressure [16], the system may have chance to approach a coherent excitonic state. Theoretical treatments beyond the Hartree-Fock approximation including fluctuation effects have identified a pseudogap opening above $T_{c}$ due to preformed excitons $[9,17]$. Application of such theories to realistic models down to low temperatures may reveal under what conditions a coherent excitonic condensate is realized in real materials.

\section{References}

[1] R. S. Knox, in Solid State Physics, Suppl. 5, edited by F. Seitz and D. Turnbull (Academic Press, New York, 1963), p.100.

[2] D. Jérome, T. M. Rice, and W. Kohn, Phys. Rev. 158, 462 (1967).

[3] L. V. Keldysh and A. N. Kozlov, JETP 27, 521 (1968).

[4] J. Eisenstein, Annu. Rev. Condens. Matter Phys. 5, 159 (2014).

[5] K. Rossnagel, J. Phys. Condens. Matter 23, 213001 (2011).

[6] Y. Wakisaka, T. Sudayama, K. Takubo, T. Mizokawa, M. Arita, H. Namatame, M. Taniguchi, N. Katayama, M. Nohara, and H. Takagi, Phys. Rev. Lett. 103, 026402 (2009). 
[7] A. Nakano, T. Hasegawa, S. Tamura, N. Katayama, S. Tsutsui, and H. Sawa, Phys. Rev. B 98, 045139 (2018).

[8] T. Kaneko, T. Toriyama, T. Konishi, and Y. Ohta, Phys. Rev. B 87, 035121 (2013)

[9] K. Seki, Y. Wakisaka, T. Kaneko, T. Toriyama, T. Konishi, T. Sudayama, N. L. Saini, M. Arita, H. Namatame, M. Taniguchi, N. Katayama, M. Nohara, H. Takagi, T. Mizokawa, and Y. Ohta, Phys. Rev, B 90, 155116 (2014).

[10] M. D. Watson, I. Marković, E. A. Morales, P. Le Fèvre, M. Merz, A. A. Haghighirad, and P. D. C. King, Phys. Rev. Res. 2, 013236 (2020).

[11] A. Subedi, arXiv:2002.08352 (2020).

[12] M.-J. Kim, A. Schulz, T. Takayama, M. Isobe, H. Takagi, and S. Kaiser, arXiv:2007.01723 (2020).

[13] E. Baldini, A. Zong, D. Choi, C. Lee, M. H. Michael, L. Windgaetter, I. I. Mazin, S. Latini, D. Azoury, B. Lv, A. Kogar, Y. Wang, Y. Lu, T. Takayama, H. Takagi, A. J. Millis, A. Rubio, E. Demler, and N. Gedik, arXiv:2007.02909 (2020).

[14] K. Okazaki, Y. Ogawa, T. Suzuki, T. Yamamoto, T. Someya, S. Michimae, M. Watanabe, Y. Lu, M. Nohara, H. Takagi, N. Katayama, H. Sawa, M. Fujisawa, T. Kanai, N. Ishii, J. Itatani, T. Mizokawa, and S. Shin, Nat. Commun. 9, 4322 (2018).

[15] T. Tang, H. Wang, S. Duan, Y. Yang, C. Huang, Y. Guo, D. Qian, and W. Zhang, Phys. Rev. B 101, 235148 (2020).

[16] Y. F. Lu, H. Kono, T. I. Larkin, A. W. Rost, T. Takayama, A. V. Boris, B. Keimer, and H. Takagi, Nat. Commun. 8, 14408 (2017).

[17] K. Sugimoto, S. Nishimoto, T. Kaneko, and Y. Ohta, Phys. Rev. Lett. 120, 247602 (2018). 